\title{
Michel Henry lecteur de Nietzsche ou le dépassement de l'hellénisme
}

\author{
Clément BERTOT \\ Université catholique de Louvain
}

Cette étude s'attachera à analyser l'enjeu des textes consacrés à Nietzsche chez Michel Henry. Les références au philosophe allemand semblent au premier abord relativement peu nombreuses, et paraissent se limiter à quelques citations éparses, ainsi qu'à certains développements parfois plus substantiels. Pourtant il est relativement aisé de remarquer que les concepts centraux de la phénoménologie henryenne peuvent être rapprochés de la pensée nietzschéenne, avec laquelle ils entretiennent des rapports implicites ou explicites. Ainsi les concepts de vie, d'affectivité, et de pulsion, qui tissent la trame de tous les textes henryens, ainsi que les notions de culture, de valeur, ou de nihilisme, plus localisées chez Henry, méritent un examen précis, afin d'évaluer s'il s'agit d'une simple résonance superficielle avec la pensée nietzschéenne, ou au contraire d'une parenté philosophique plus profonde.

Un simple survol permet de constater que les références à Nietzsche concernent un nombre restreint de textes, essentiellement La naissance de la tragédie et, dans une moindre mesure, La généalogie de la morale. Henry ne travaille pas le corpus nietzschéen dans son intégralité. Il sélectionne certains textes et concepts déterminants, afin d'interroger leurs ressources phénoménologiques. Nietzsche est une figure chez Henry, et plus précisément une référence stratégique, qui vise à mettre en place un certain geste polémique à l'égard des autres phénoménologues, et d'abord à l'égard de la tradition occidentale dont ils sont les héritiers. Selon nous, c'est l'enjeu d'une approche non grecque de la phénoménalité qui caractérise la lecture henryenne de Nietzsche. Les présupposés phénoménologiques fondamentaux de la pensée grecque, qui gouvernent tout le développement de la philosophie occidentale, fournissent à la phénoménologie historique un horizon prédéterminé de questionnement, dont la phénoménologie matérielle entend dépasser les déficiences. Nietzsche est tout particulièrement l'auteur qui sert de point d'appui à Henry pour remettre en question cette tradition, davantage que Biran ou Schopenhauer, en tant que les avancées décisives de leurs philosophies restent malgré tout déterminées par des aspects fondamentalement grecs.

On peut d'emblée s'étonner d'un tel usage de la pensée nietzschéenne, dès lors qu'on se souvient combien Nietzsche a pu valoriser la Grèce dans ses écrits. Toutefois Nietzsche accorde sa préférence à une certaine Grèce, et en critique une autre. Schématiquement, le philosophe allemand remet en question la Grèce 
classique, celle des philosophes, la Grèce idéaliste de Socrate et de Platon, pour faire retour à la Grèce tragique de l'époque archaïque. Aux yeux de Henry, Nietzsche constituerait une exception dans la philosophie occidentale, en tant qu'il mettrait en place des présupposés phénoménologiques différents de ceux de la Grèce idéaliste. Il convient selon nous de lire les textes de Henry consacrés à Nietzsche selon le fil conducteur de cette stratégie anti-grecque. D'un point de vue synoptique, la relation à Nietzsche pourra toutefois être divisée en deux grandes périodes, d'abord une période de référence positive à Nietzsche, puis une seconde période dans laquelle Henry prendra ses distances avec le philosophe allemand.

La première période est consacrée à l'élaboration d'une lecture phénoménologique de Nietzsche, dont l'objectif consiste à imprimer une orientation nietzschéenne à la phénoménologie, selon la stratégie anti-grecque que venons d'identifier. Cette lecture de Nietzsche est amorcée dès L'Essence de la manifestation, même si les références à Nietzsche restent encore discrètes. Au fil des œuvres, les textes consacrés à Nietzsche se font plus nombreux, pour culminer dans ce que nous appellerons le « moment nietzschéen » de la pensée de Henry, qui regroupe les deux publications de 1985 et 1987, La Généalogie de la psychanalyse et La Barbarie. Ces deux œuvres jouent un rôle stratégique majeur dans le dispositif anti-grec de Henry. Suite à ce moment nietzschéen, une seconde période est amorcée, dans laquelle les références au philosophe allemand se font plus rares, pour devenir de plus en plus critiques. Cette période correspond chez Henry à la mise en place d'une philosophie du christianisme, c'est-à-dire au développement d'un modèle de pensée qui viendra concurrencer le modèle grec. Henry et Nietzsche, deux penseurs de la vie, consacrent tous deux leur dernière philosophie au christianisme, mais selon des logiques bien évidemment antithétiques. Nietzsche écrit L'Antéchrist, sous-titré «Essai de critique du christianisme », et Henry publiera quant à lui C'est moi la Vérité, sous-titré «Pour une philosophie du christianisme ». L'adhésion initiale à Nietzsche, déterminée par un rejet de la Grèce, sera suivie et remplacée par une philosophie du christianisme, devenue incompatible avec la pensée de l'auteur de l'Antéchrist. À partir du repérage de ces deux périodes, le problème qui sera traité dans cette étude est d'une grande simplicité. Quelle conception de la vie a d'abord rapproché Henry de Nietzsche, avant de l'en séparer ? Notre propos sera divisé en deux parties. Nous analyserons d'abord l'appropriation de la pensée nietzschéenne dans la phénoménologie anti-grecque de Henry, avant d'étudier l'orientation antinietzschéenne de la philosophie henryenne du christianisme. 


\section{La stratégie nietzschéenne de Michel Henry : penser contre la Grèce}

\subsection{Une interprétation phénoménologique de La naissance de la tragédie}

Dans un article intitulé « Michel Henry et l’Hellénisme », Gilbert RomeyerDherbey rappelle la nature des présupposés phénoménologiques fondamentaux issus de la philosophie grecque depuis Parménide : «La pensée grecque est une pensée solaire [...]. Son emblème, c'est Apollon, le dieu qui darde ses rayons dans le ciel ouvert. " ${ }^{1}$ La référence à cette divinité, à laquelle Nietzsche aura su conférer un statut philosophique inédit, permet à Henry de désigner un certain mode de la phénoménalité, comprise comme transcendance ou extériorité, comme venue dans la lumière. En quoi ce paradigme est-il caractéristique de la Grèce ? En quoi est-il au principe de la tradition philosophique, comme ce qui la commence et la commande, jusque dans ses développements ultimes en phénoménologie ?

Selon Henry, la Grèce apollinienne renvoie à une compréhension limitée de la phénoménalité. De quoi ce paradigme grec de la phénoménalité comme extériorisation dans la lumière est-il l'oubli ? En tant que pensée solaire, la philosophie grecque se caractérise par un primat de la visibilité, et par l'ignorance de l'intériorité invisible. Plus largement, dans la tradition issue de la Grèce, tout ce qui se montre apparaît dans un horizon d'extériorité, qui n’est autre que le monde, compris en un sens phénoménologique. Apollon est l'emblème des apparitions qui s'exhibent en plein jour, et cette phénoménalité diurne passe sous silence l'affectivité orgiastique de Dionysos, celle de la joie et de la souffrance qui échappent à toute lumière. D’après Henry, la philosophie grecque, en développant sa logique sous les auspices d'Apollon et du sanctuaire de Delphes, n'a pas su reconnaître l'intériorité affective dans sa spécificité. La pensée henryenne se constituera dès lors contre le "primat ontologique de la transcendance », qui gouverne la «pensée philosophique depuis son origine hellénique » ${ }^{2}$. Au sein de la tradition philosophique, la figure de Nietzsche constitue alors une exception pour le phénoménologue français. Dès son premier grand texte philosophique, Nietzsche mettrait en effet en place des présupposés phénoménologiques différents de ceux de la pensée grecque «apollinienne », pour mieux faire retour à ce qui la féconde secrètement.

Il convient de rappeler que La naissance de la Tragédie fait référence à Apollon comme à l'emblème d'une pulsion artistique, celle qui produit les belles formes de l'art plastique. La statuaire grecque est issue de la pulsion apollinienne. Mais Henry

\footnotetext{
${ }^{1}$ Gilbert Romeyer-Dherbey, Michel Henry. Pensée de la vie et culture contemporaine, Paris, Beauchesne (coll. « Prétentaine »), 2006, p. 38.

${ }_{2}^{2}$ Michel Henry, EM, p. 56-57.
} 
a raison de souligner que le résultat de cette création artistique est bien un certain type de manifestation, au sens phénoménologique, celle de la splendeur des formes visibles des statues, qui sont de pures surfaces dénuées d'intériorité. Prenant le contrepied de cette compréhension de la phénoménalité, Henry confère à $L a$ naissance de la tragédie un statut phénoménologique capital. Selon lui ce texte n'est rien moins que l'acte inaugural de la mise en place d'un modèle alternatif de la phénoménalité en philosophie, qui viendrait révéler les insuffisances des présuppositions phénoménologiques de la tradition issue de la Grèce apollinienne, pour retrouver ce qui la détermine en profondeur. En donnant un statut philosophique à la figure de Dionysos, Nietzsche serait parvenu à dégager une phénoménalité souterraine, celle de l'auto-impression de la vie. Nietzsche a luimême souligné à plusieurs reprises que l'origine de Dionysos n'était pas grecque ${ }^{3}$. Dionysos est une divinité assimilée par les grecs, mais son origine se trouve en Inde et en Mésopotamie. Henry aura su donner un sens phénoménologique à cette origine barbare.

Ce statut phénoménologique de l'affectivité permet d'abord à Henry de mettre en place un modèle de phénoménalité concurrent d'un autre, mais plus encore, avec Nietzsche, Henry trouve l'occasion de hiérarchiser ces deux modes. Dionysos, tout en étant invisible, est plus réel qu'Apollon, et si ce dernier se caractérise par la lumière, il déploie une "phénoménalité précaire " ${ }^{4}$, entachée d’irréalité. Face à la phénoménalité nocturne de Dionysos, celle de la l'auto-impression de la vie, Apollon fait pâle figure. La luminosité du monde ne peut se donner que sur fond de cette révélation orgiastique et ténébreuse. La faiblesse phénoménologique d'Apollon permet à Henry de reprendre la caractérisation nietzschéenne de la conscience diurne comme rêve, analysée dans le premier paragraphe de La naissance de la tragédie. La représentation, comme un rêve, glisse à la surface des choses. Henry réinvestit la notion nietzschéenne d'« apparence », pour penser la précarité ontologique des apparitions du monde.

Cette hiérarchie étant posée, il convient de dissiper un malentendu possible. Lorsque Henry évoque la pensée de Nietzsche comme une "pensée solaire " ${ }^{5}$, « éclatante " ${ }^{6}$, comme une "pensée radieuse " ${ }^{7}$, il ne s'agit en rien d'assimiler le philosophe allemand aux présupposés phénoménologiques issus de la Grèce apollinienne. Chez Henry, l'éclat de la pensée nietzschéenne renvoie moins à la lumière qu'au feu. Henry rend ainsi hommage aux «figures éblouissantes de la vie que sont les forts, les nobles, les animaux ${ }^{8}$, en soulignant qu'à leur contact «le

\footnotetext{
${ }^{3}$ Cf. Friedrich Nietzsche, La naissance de la tragédie, trad. par P. Wotling, Paris, Le livre de poche (coll. « Les Classiques de la Philosophie »), 2013, § 20 et 21.

${ }^{4}$ Michel Henry, GP, p. 329.

${ }^{5}$ Ibid.

${ }^{6}$ Ibid., note 85, p. 330.

${ }^{7}$ Ibid., p. 9.

${ }^{8}$ Ibid., p. 10.
} 
texte nietzschéen s’enflamme », que « les images crépitent », que " chaque preuve est un brasier » ${ }^{9}$. Il est ici question d'une affectivité brûlante, qui constitue le fond à partir duquel toute lumière prend naissance : «Plus intense [...] est l'expérience que la vie fait d'elle-même [...], plus vives, plus lumineuses sont les images où elle se projette. ${ }^{10}$ La brillance d'Apollon est alors pensable comme une incandescence issue du pathos dionysiaque soustrait à toute lumière. Plus la vie s'affecte souterrainement, plus elle rend le monde éclatant. Apollon devient une « ombre » ${ }^{11}$ de Dionysos, alors même qu'il est surnommé « le brillant ».

La pensée de Nietzsche joue alors un rôle déterminant chez Henry. Elle permet de penser le lien de conditionnement entre les deux modes de la phénoménalité qui traversent toute son œuvre. La vie a besoin d'extérioriser son pathos dans un monde pour se libérer d'un trop plein affectif. Il s'agit alors de caractériser génétiquement l'affectivité de la représentation par le recours à la notion d'Imago ${ }^{12}$. Chez Nietzsche, il ne s'agit plus de « représentation » au sens strict. Le monde apparaît au sein d'une projection de la vie qui jette au dehors son propre pathos dans des images, en leur conférant telle ou telle valeur, selon les déterminations à chaque fois singulières de l'affectivité. La notion schopenhauerienne de représentation est dépassée et privée de son autonomie. C’est également la division inintelligible, chez Schopenhauer, de la volonté et de la représentation, qui est surmontée avec Nietzsche ${ }^{13}$. Le monde devient un pouvoir de la vie, qui précède toute extériorité, et la rend possible. Cette démarche généalogique, dans laquelle la primauté de la vie par rapport au monde est retrouvée, va jouer en retour un rôle crucial dans l'enquête henryenne visant à expliquer l'oubli de la vie qui caractérise la tradition issue de la Grèce. Comment l'extériorité du monde pouvait-elle être présentée comme autosuffisante, et comme exclusive de tout autre mode de phénoménalité ? Comment ce qui est dérivé pouvait-il être posé comme premier et auto-suffisant ? C'est par la réappropriation de la démarche nietzschéenne de la généalogie que Henry tentera d'élucider cet impensé de la phénoménologie.

\footnotetext{
${ }^{9}$ Michel Henry, PV-II, p. 151.

${ }^{10}$ Michel Henry, EM, p. 329.

${ }^{11}$ Ibid., p. 330.

${ }^{12}$ Cf. Michel Henry, GP, p. 313 et 327.

${ }^{13}$ Cf. Ibid., p. 312-313.
} 


\section{2. " De la Grèce à Heidegger " : la fonction généalogique de Nietzsche dans une contre-histoire de l'être}

Selon Henry, Heidegger est l'adversaire phénoménologique incarnant à l'extrême la promotion de la phénoménalité grecque. On retrouve souvent, sous la plume de Henry, l'expression suivante : "de la Grèce à Heidegger " ${ }^{14}$. Cette expression ne désigne pas seulement la présence diachronique de présupposés phénoménologiques identiques. Elle permet également d'identifier une élaboration ultime de ces présupposés dans la pensée heideggérienne. Le phénoménologue allemand élabore, avec une puissance philosophique inégalée, une compréhension de la phénoménalité comme monde, le phénomène étant compris comme venue dans la lumière ${ }^{15}$. Par la lecture originale de Nietzsche qu'il développe, Henry entre en réalité en confrontation avec le phénoménologue allemand. D’une part, Henry critique l'interprétation heideggérienne de Nietzsche. Cette lecture fait autorité dans le courant phénoménologique, à tel point qu'aucune autre interprétation d'envergure n’a été tentée. L'absence de confrontation à Nietzsche, chez les phénoménologues français «heideggériens », pourrait être interprétée comme un ralliement implicite aux deux tomes du Nietzsche. On ne trouve en effet que très peu de mentions de Nietzsche chez Sartre, Levinas, ou Merleau-Ponty, comme si le sort de la pensée nietzschéenne avait été scellé par Heidegger. Dans ce contexte strictement français, la lecture de Henry ne peut pas être analysée indépendamment de l'éclairage de ce rapport polémique à Heidegger. Henry s'attache d'une part à extraire Nietzsche de l'interprétation heideggérienne, mais plus profondément, Nietzsche est un recours utilisé par Henry contre la pensée heideggérienne dans sa globalité, pas seulement contre les textes heideggériens consacrés à Nietzsche.

La Généalogie de la psychanalyse mettra en place une stratégie tout à la fois antiheideggérienne et anti-grecque, en réinvestissant une démarche philosophique nietzschéenne. Le titre de l'ouvrage de 1985 est une référence évidente à $L a$ généalogie de la morale. Chez Nietzsche, la généalogie désigne l'enquête régressive qui cherche à identifier les pulsions comme sources des interprétations, par un retour à la vie comme origine des valeurs. La démarche henryenne relève-t-elle d'un tel modèle ? La réponse à cette question exige de développer une analyse précise. Henry cherche à établir la généalogie d'une certaine compréhension de la phénoménalité. Le titre de l'ouvrage peut alors prêter à confusion. Il s'agit moins de s'intéresser à la psychanalyse qu'à la tradition philosophique dont elle est le résultat. La psychanalyse, comme négation de tout apparaître autre que celui de la

\footnotetext{
${ }^{14}$ Cf. par exemple Michel Henry, AD, p. 67.

${ }^{15}$ Cf. Ibid., p. 29 : «C'est chez Heidegger que l'apparaître du monde est conduit à son plus haut degré d'élaboration. Dans le paragraphe 7 de Sein und Zeit, le phénomène est compris au sens grec — phainomenon - à partir de la racine pha, phos, qui signifie la lumière, en sorte qu'apparaître signifie venir dans la lumière, la clarté ».
} 
conscience, n'est pas un commencement, mais un aboutissement. Le sous-titre de l'ouvrage, "le commencement perdu », indique que la vie est d'emblée manquée dans la tradition philosophique issue de la Grèce, autant que dans la discipline psychanalytique, puisqu'au sein de cette dernière l'inconscient est le faux nom de la vie, frappée de non-apparaître. C'est par la prise en compte de ce manquement qu'on pourra expliquer généalogiquement, et à nouveaux frais, l'histoire de la philosophie, et le conditionnement qu'elle exerce sur la démarche psychanalytique. En faisant de Nietzsche le représentant ultime des métaphysiques de la représentation, Heidegger ne parvient pas à reconnaître la vie nietzschéenne comme un mode d'apparaître non représentatif, et révèle ainsi ses présupposés phénoménologiques fondamentaux, ceux dont la psychanalyse est également issue, à savoir les présupposés grecs. Dès lors, la généalogie de la Grèce pourra être accomplie le plus efficacement comme une généalogie de l'histoire heideggérienne de l'être, ou comme généalogie de la psychanalyse.

La démarche généalogique mise en place par Henry est un geste d'inspiration nietzschéenne, mais sa nature propre se démarque du modèle strictement nietzschéen. Ce qui est commun aux deux généalogies, c'est de remettre en cause l'autonomie de ce que la tradition pose comme un fondement auto-suffisant, les valeurs chez Nietzsche, ou le monde chez Henry. Chez Nietzsche, les valeurs sont reconduites à la vie et à ses affects, comme instances souterraines opérant des choix de valorisation et de dévalorisation. Chez Henry, le monde est reconduit à la vie comme instance génératrice au point de vue phénoménologique. La critique de l'autonomie de la valeur chez Nietzsche ne reconduit pas à un nouveau fondement, mais à une logique de la mesure ${ }^{16}$. En revanche, chez Henry, la vie est posée comme fondement, au sens phénoménologique : elle est l'auto-donation dont dérive toute donation possible, et plus particulièrement celle du monde. Le perspectivisme axiologique de Nietzsche laisse place, chez Henry, à un donné premier, à un absolu. La Généalogie de la psychanalyse met en place discrètement ce que nous choisissons d'appeler une contre-histoire de l'être, fondée sur une démarche généalogique d' « inspiration » nietzschéenne, comme reconduction à la vie. Il s'agit de remettre en cause l'histoire heideggérienne de l'être par l'absolu dont elle est l'oubli, qui détermine également la psychanalyse et ses méthodes. Henry bâtira son histoire de la métaphysique autour d'un oubli, à l'instar de Heidegger, mais pour montrer que l'oubli de la vie est plus profond que l'oubli de l'être. L'histoire heideggérienne de l'être dessinait une gradation. Dans les Temps modernes, l'oubli de l'être est croissant de Descartes à Nietzsche, puisque la subjectivité s'arroge de plus en plus les prérogatives de l'être, occultant ce dernier en le réduisant à l'ob-jet de la représentation, comprise comme certitude, puis comme volonté de puissance. Aux yeux de Heidegger, l'oubli de l'être s'affirmerait avec Nietzsche dans le

${ }^{16}$ Cf. à ce sujet notre étude "Connaissance et généalogie chez Nietzsche », dans L'art du comprendre, numéro 24, 2015, p. 99-122. 
triomphe de la subjectivité, et la considération de l'étant comme pur objet de maitrise.

Selon Henry, une gradation inverse se dessine de Descartes à Nietzsche : l'être n'est pas oublié graduellement, il s'affirme progressivement comme vie. Chez Descartes a lieu une première réduction ${ }^{17}$, celle de la représentation, de sorte que tout ob-jet est jeté hors du domaine d'une certitude possible, et frappé de nullité par le doute. La réduction de la représentation reconduit à un commencement inébranlable, à une impression immédiate, comme sphère absolue d'apparaître. Cet apparaître est un sentir qui n'est autre que l'apparaître de l'apparaître, la vie, ou l'être. Descartes dit bien «je suis». Avec Nietzsche a lieu une réduction plus radicale encore, qui n'est plus théorique mais existentielle : elle «n'est plus le doute mais le désespoir $"{ }^{18}$. Henry évoque en effet, avec tout l'étonnement que peut susciter une telle expression d'un point de vue strictement nietzschéen, le «cogito radical de Nietzsche ${ }^{19}$, issu de la tentative avortée de la vie de se fuir elle-même lorsqu'elle se trouve dans la souffrance et la faiblesse, démarche qui aboutit paradoxalement à réaffirmer le lien indissoluble de la vie avec elle-même. Le cogito nietzschéen, plus radical encore que celui de Descartes, se formule par la proposition «je suis ce que je suis » (Ich bin, der ich bin). Henry emprunte cette formule à La généalogie de la morale ${ }^{20}$, afin de caractériser la souffrance nietzschéenne comme un cogito au sens phénoménologique. Cette interprétation ne vise pas seulement à remettre en cause la lecture heideggérienne de Nietzsche, qui considérait la pensée du philosophe allemand comme le point culminant de l'oubli de l'être. Plus profondément, la stratégie nietzschéenne permet de remettre en cause la conception heideggérienne de l'être, comme conception grecque de l'être. Nous retrouvons ici l'enjeu central de notre démonstration. Heidegger est le philosophe de la question de l'être, mais il manque son sens profond, en restant dans un horizon de questionnement prédéterminé, un horizon grec. Le cogito nietzschéen identifié par Henry n'est rien moins que l'être venant à lui-même comme vie, selon une signification anti-grecque. L'être s'auto-affecte, et s'auto-atteste dans le cogito radical de Nietzsche, et en ce sens la phénoménologie matérielle ne remet pas en cause la pertinence philosophique de la question de l'être ${ }^{21}$. Elle la pose à nouveau frais, et vient résoudre ses difficultés.

En effet, le recours à Nietzsche, chez Henry, permet de surmonter la fragilité qui pèse sur l'être heideggérien, tel qu'il est thématisé notamment par le

\footnotetext{
${ }^{17}$ Michel Henry, GP, p. 100.

${ }^{18}$ Ibid., p. 298.

${ }^{19}$ Ibid., p. 297.

${ }^{20}$ Cf. Friedrich Nietzsche, La généalogie de la morale, III, § 14, trad. par P. Wotling, Paris, Le livre de poche (coll. « Les Classiques de la philosophie »), 2000.

${ }^{21}$ C'est du moins vrai à cette époque de la pensée de Henry. Dans les textes plus tardifs sur le christianisme, le concept d'être sera abandonné au profit de la notion de l' «advenir ». Cf. par exemple Michel Henry, MV, p. 74.
} 
phénoménologue allemand dans la Lettre sur l'humanisme. L'être est vulnérable, il cherche un garde en l'homme ${ }^{22}$, et c'est pour montrer que l'« être se garde d'abord lui-même $"{ }^{23}$ que Henry fait retour à Nietzsche. Les deux caractéristiques fondamentales de l'apparaître de la vie nietzschéenne identifiées par Henry, l'immanence et l'affectivité ${ }^{24}$, s'opposent ainsi à l'être heideggérien dans une logique d'autonomisation. Le repérage de ces deux caractéristiques ouvre le chapitre VII de la Généalogie de la psychanalyse, et commande toute la lecture henryenne de Nietzsche, afin de substituer l'immanence à l'extériorité heideggérienne, et l'affectivité à la réceptivité. Il s'agit bel et bien de rendre à l'être une capacité d'apparaître autarcique.

Au final, Henry se revendique de Nietzsche pour penser l'être comme subjectivité, contre la lecture heideggérienne qui associait la métaphysique de la subjectivité à l'oubli de l'être. Le sujet nietzschéen est « ce qui, faisant apparaître l'apparaître, fait du même coup être tout ce qui est ${ }^{25}$. Aux yeux de Henry, Heidegger conçoit la subjectivité nietzschéenne de façon superficielle. Il n’a pas su lui accorder un sens ontologique, comme surgissement initial de l'Être, comme «irruption triomphante de la révélation et $[\ldots]$ première victoire sur le néant ${ }^{26}$. Il en va ici d'une structure interne de l'être comme immanence phénoménologique. Là encore, on peut s'étonner, d'un point de vue strictement nietzschéen, que le philosophe allemand soit présenté comme un penseur de la subjectivité ainsi que de l'être, dès lors qu'on sait combien il a critiqué ces notions, mais Henry entend dépasser l'orthodoxie nietzschéenne pour interroger la fécondité phénoménologique de sa pensée.

Cette ligne d'argumentation autour de la structure interne de l'être s'appuie sur une remise en question de l'interprétation heideggérienne de la notion de volonté de puissance. En déterminant la volonté de puissance comme « volonté de volonté »" Heidegger impose au concept central du nietzschéisme un fonctionnement qui relève de la représentation, ce qui suppose de diviser la volonté en deux instances : d'une part un sujet qui veut, et d'autre part un objet qui est voulu. En interprétant explicitement la pensée nietzschéenne comme une métaphysique de la représentation, et comme la manifestation ultime de l'oubli de l'être, Heidegger trahit son incapacité à penser la volonté de puissance comme structure interne de l'être, et confesse ainsi plus généralement aux yeux de Henry son incapacité à penser l'être. Henry entend redonner un sens ontologique à la volonté de puissance, aux antipodes de l'interprétation heideggérienne qui entendait la réduire à un simple processus ontique. A la détermination heideggérienne de la volonté de puissance

\footnotetext{
${ }^{22}$ Michel Henry, GP, p. 107-123.

${ }^{23}$ Ibid., p. 116.

${ }^{24}$ Ibid., p. 249.

${ }^{25}$ Michel Henry, PV-II, p. 21.

${ }^{26}$ Ibid., p. 130.

27 Cf. Martin Heidegger, Nietzsche II, trad. par P. Klossowski, Paris, Gallimard (coll. « Bibliothèque de la philosophie »), 1971, p. 215.
} 
comme volonté de volonté, Henry oppose sa propre interprétation de la volonté de puissance, comme puissance de la volontée ${ }^{28}$. Il y a une incoercibilité du lien qui lie toute puissance à elle-même, de sorte qu'une hyperpuissance précède toute volonté, la plus faible comme la plus forte, en la contraignant d'être ce qu'elle est, d'être le soi de l'être ou l'être comme soi, c'est-à-dire la subjectivité. Henry transgresse ici les enjeux strictement nietzschéens de la notion de volonté de puissance, puisque chez Nietzsche ce concept n'est jamais présenté dans une signification ontologique à strictement parler ${ }^{29}$, mais l'interprétation henryenne a le mérite de dégager la fécondité phénoménologique de la notion, contre son appauvrissement heideggérien qui entendait la réduire à une dimension métaphysique.

In fine, l'enjeu de cette lecture de Nietzsche consiste à développer une pensée nongrecque de l'être, afin d'ôter au principal concept de la pensée grecque son sens grec. Cette lecture théorique aura des enjeux pratiques, qui concerneront la modernité, et seront inspirés, là encore, par la pensée nietzschéenne. Henry réfléchit à la conceptualisation du sens pratique d'une subjectivité ontologique dont la modernité comme nihilisme est la négation. Mais c'est aussi autour de cet enjeu pratique que la rupture avec Nietzsche aura lieu, comme nous allons le voir dans une seconde partie.

\section{La modernité selon Nietzsche et Henry}

\subsection{La critique du nihilisme chez Henry : une posterité nietzschéenne ?}

En prenant en charge le problème de la modernité, la pensée henryenne réinvestit un cadre de pensée nietzschéen. L'interrogation de la modernité comme crise, chez les phénoménologues, s'inscrit le plus souvent dans un cadre de pensée husserlien. Nous voudrions montrer que c'est la perspective nietzschéenne qui domine l'interprétation henryenne. La méditation sur la modernité conduira toutefois le phénoménologue français à rompre avec Nietzsche, par la mise en place d'une philosophie du christianisme dans la dernière période de sa production intellectuelle. Avant d'analyser cet enjeu, il convient de montrer en quoi la compréhension de la modernité chez Henry est issue de la philosophie nietzschéenne. Selon nous, c'est la perspective d'une compréhension anti-grecque de la subjectivité qui commande cette lecture.

La critique de la conception «grecque » de l'être est en réalité subordonnée à la remise en question de l'humanisme métaphysique issu de la Grèce. Si l'être est vie,

\footnotetext{
${ }^{28}$ Michel Henry, GP, p. 256.

${ }^{29}$ Nous renvoyons ici au paragraphe 36 de Par-delà bien et mal, et à l'interprétation de ce texte dans le livre de Patrick Wotling, Nietzsche et le problème de la civilisation, Paris, PUF (coll. « Quadrige »), 1995, Première partie, chapitre 2, «L’hypothèse de la volonté de puissance », p. 5182.
} 
l'homme n'est rien d'autre que cette vie comme intériorité pathétique. L' « homme », au sens d'une essence séparée, est une notion dépourvue de pertinence phénoménologique. Henry rejoint ici la critique nietzschéenne de l'anthropocentrisme métaphysique. La reconnaissance d'un privilège métaphysique de l'homme continue à nourrir souterrainement la pensée de Heidegger, et trouve en elle son aboutissement, dans le concept du Dasein. Henry critique en effet l'ontologie privative de la vie exposée dans le paragraphe 10 de Être et temps, en adoptant là encore une stratégie nietzschéenne. Heidegger reste prisonnier du préjugé humaniste qui traverse toute la pensée occidentale depuis les grecs. Le Dasein est quelque chose d'autre qu'un vivant, quelque chose de plus. Chez Heidegger, la vie doit dès lors se comprendre «par une sorte de soustraction par rapport à ce que nous sommes » ${ }^{30}$, selon la juste formule de Henry. La vie est pensée par Heidegger comme la privation de certains privilèges du Dasein. La vie est le Dasein «moins » quelque chose. C'est pourquoi Henry s'attache à souligner que, chez Heidegger, le Dasein est « plus qu'un vivant » ${ }^{31}$.

Contre ce dispositif heideggérien, Henry se revendique de Nietzsche pour penser un modèle inverse, un modèle délivré de l'humanisme métaphysique de la Grèce. Il s'agit de placer méthodologiquement l'animalité comme forme première, non altérée de la vie, comme immanence radicale, afin de déterminer le statut de l'homme selon un statut dérivé au regard de ce dispositif initial. L’homme est une tentative de la vie pour s'arracher à l'immanence. Il est une maladie de la vie. Henry retravaille un concept central de la pensée nietzschéenne, celui de l'homme comme animal malade, pour remettre en cause l'ontologie privative de Heidegger. Ce qui est en jeu ici relève d'un nouveau commencement de la philosophie, qui fonctionne comme une inversion de la thèse heideggérienne. Selon Henry, l'homme est la vie « moins » quelque chose. La vie n'est pas ici le résultat d'une soustraction comme chez Heidegger, mais c'est l'homme qui est caractérisé comme celui qui tente de se soustraire à la vie. On commettrait une lourde erreur à lire, sans nuances, la pensée de Henry comme une simple philosophie de l'immanence. Henry tente plutôt de penser l'énigme de l’arrachement à l’immanence, la rupture désespérée, avortée, vouée à l'échec, de la vie avec elle-même.

Le cogito radical de Nietzsche a prouvé que le lien de la vie à elle-même était indissoluble. Mais la tentative en permanence renouvelée pour briser ce lien de la vie avec elle-même caractérise bel et bien l'homme, et vient le définir, ou plutôt lui conférer un statut phénoménologique propre, par une sorte de paradoxe interne : « L'autodestruction impossible de l'essence intérieure de la vie, autodestruction qui, comme telle, n'en finit pas, c'est ce que Nietzsche appelle la maladie de la vie, ce qui fait de l'homme — en tant que l'immanence est l'animalité et ce qu'il s'agit

\footnotetext{
${ }^{30}$ Michel Henry, AD, p. 130.

${ }^{31}$ Ibid.
} 
d'ébranler — un "animal malade". ${ }^{32}$ Ce texte — absolument stupéfiant — permet à Henry de dessiner, à partir de Nietzsche, une anthropologie phénoménologique, sans recourir au concept d'une essence humaine, encore moins à une pensée de type anthropologique au sens strict. L’homme est caractérisé phénoménologiquement comme une anomalie de l'essence. Cette auto-négation de la vie ne doit pas être comprise au sens d'une destruction de la vie biologique, mais au sens d'une négativité interne à la vie phénoménologique. Dans la plénitude de sa donation à soi, la vie ne peut se supporter elle-même, mais ne pouvant se soustraire effectivement à elle-même, elle se retourne contre elle-même en son propre sein. Le nihilisme vient alors définir le mouvement par lequel la vie tente d'échapper à sa propre phénoménalisation, à son propre souffrir, afin de ne plus s'affecter ${ }^{33}$. La possibilité d'une négation immanente à la vie est un paradoxe, et dans ce cadre, Nietzsche est le seul penseur capable de fournir une base conceptuelle à Henry pour thématiser une telle énigme.

Dans la Barbarie, texte incontestablement le plus nietzschéen de Henry, l'analyse du nihilisme vient caractériser la modernité, et plus précisément son dispositif scientifique et technique. Les immenses progrès de la science sont indéniables, mais l'idéologie réduisant tout savoir à celui de la science remet en cause la connaissance immédiate que nous avons de nous-mêmes et du monde en tant que vivants. La science exclut la vie de l'ontologie. Cette vie ontologique et phénoménologique est le sol des idéalisations de la science, mais la science nie ce sol préalable dont elle provient. C'est ce mouvement paradoxal, de négation de soi par soi, que Henry nomme " barbarie ». Cette dernière est l'aboutissement des présuppositions phénoménologiques de la Grèce, et de l'homme théorique dont Nietzsche entendait déjà développer la critique dans La naissance de la tragédie. La barbarie n'est pas la sauvagerie, elle ne renvoie pas à la destruction de la vie biologique. Elle suppose au contraire un haut degré de culture, dont elle est pourtant la négation. Le titre de l'ouvrage de 1987 entre étonnement en résonnance avec un texte de Nietzsche issu de Ecce homo, dans lequel le philosophe allemand faisait retour sur le projet de la Seconde Inactuelle, consacrée au développement de la science : "La deuxième Intempestive (1874) met en évidence ce qu'il y a de dangereux, de rongeant et de toxique, dans notre manière de pratiquer la science - : la vie malade [...]. Le but, qui est la civilisation, se perd : - le moyen, la pratique moderne de la science, barbarise... ${ }^{34}$

Barbarie nietzschéenne, barbarie henryenne. Henry retravaille l'opposition entre science et culture déjà relevée par Nietzsche. Il faut rappeler, même si ce point est bien connu, que la pensée du philosophe allemand n'est pas dominée par l'enjeu de

\footnotetext{
${ }^{32}$ Michel Henry, PV-II, p. 154.

${ }^{33}$ Cf. Ibid.

${ }^{34}$ Friedrich Nietzsche, Ecce homo, trad. par E. Blondel, Paris, GF Flammarion, 1992, « Pourquoi j'écris de si bons livres », Les Intempestives, § 2.
} 
la vérité, mais par un enjeu pratique, celui de la culture, et par la figure du philosophe comme médecin de la civilisation. C'est dans le sillage de cette orientation de la pensée nietzschéenne que se positionne La Barbarie. Henry réinvestit la démarche nietzschéenne d'une critique philosophique de la science, élaborée dans La naissance de la tragédie, et plus encore dans les Inactuelles. Le travail philosophique de Nietzsche consistait à cette époque à positionner la philosophie dans une relation hiérarchique à l'égard de la science ${ }^{35}$. Le philosophe médecin ne doit pas viser l'augmentation indéfinie de la connaissance, contrairement au scientifique, mais doit déterminer la valeur du développement de la science dans l'équilibre général de la vie. Aux yeux de Henry, l'excès de science, au détriment d'autres formes de vie haute, à savoir l'art, la religion, l'éthique, fait tomber en désuétude ces modalités de la culture comme des illusions nonscientifiques. Le combat entre science et culture devient la gigantomachie du monde moderne.

Henry s'approprie le concept nietzschéen de culture pour lui donner un sens phénoménologique. La culture est une auto-révélation se caractérisant par un impératif d'auto-accroissement. Dans La barbarie, Henry identifie explicitement cette notion à la volonté de puissance ${ }^{36}$. La culture est d'abord une praxis interne dans laquelle la vie vient davantage en elle-même, pour s'affecter plus intensément. La culture n'est pas réductible à des œuvres extérieures. Ainsi, dans l'art, la sensibilité veut par exemple sentir davantage. Il est question de cet autoaccroissement phénoménologique dans tous les domaines de la culture, notamment dans l'éthique et la religion, selon des modalités à chaque fois spécifiques. Le mouvement du nihilisme, dont l'idéologie de la science est le fondement, vient rompre ce procès phénoménologique d'auto-accroissement. Cette compréhension henryenne de la modernité, très largement inspirée par Nietzsche, sert de nouveau à remettre en question la pensée heideggérienne. Le phénoménologue allemand se donnait déjà pour tâche d'analyser les conséquences de l'ontologie grecque sur la modernité, mais son questionnement restait prisonnier d'un horizon grec. Ce point est particulièrement sensible dans l'ignorance de la critique nietzschéenne de la science dont Heidegger se rend coupable. En interprétant la pensée de Nietzsche comme l'aboutissement, dans les Temps modernes, de la domination de la science et de la technique sur la nature, Heidegger croit pouvoir accuser Nietzsche d'avoir levé les dernières barrières qui empêchaient encore l'homme de devenir le Seigneur de la Terre. Selon Heidegger, la pensée nietzschéenne accomplirait le nihilisme en ouvrant l'époque du dévoilement de tout étant comme fond disponible en vue de l'homme. Nietzsche serait par excellence le penseur de l'animal rationnel grec, et les

\footnotetext{
${ }^{35}$ Friedrich Nietzsche, Considérations Inactuelles I et II et Fragments posthumes 1872-1874, trad. par P. Rusch, Paris, Gallimard, 1990, FP 19 [41], p. 186-187.

${ }^{36}$ Michel Henry, B, p. 175.
} 
implications de cet anthropocentrisme métaphysique seraient poussées jusqu’à leurs ultimes conséquences dans le dispositif scientifique et technique de la modernité.

Contre cette lecture, Henry oriente sa propre critique de la modernité en faisant jouer à Nietzsche un rôle contraire. La critique nietzschéenne de la science permet de définir le nihilisme comme une négation de la vie, une négation de la subjectivité ontologique, et c'est précisément ce point qui est manqué par Heidegger dans sa propre pensée du nihilisme. Le dispositif de La barbarie est nietzschéen dans le sens où l'homme n'est plus pensé par Henry comme l'animal rationnel, mais d'abord comme vie. La science — plus précisément l'idéologie qui en est issue — n'est pas l'accomplissement des facultés naturelles de l'homme, elle est au contraire une négation immanente à la vie. Dans ses dernières œuvres, Henry conférera à la philosophie du christianisme un rôle déterminant dans la lutte contre le nihilisme, et sur ce point précis, Nietzsche cessera d'être un allié contre Heidegger et la Grèce.

\subsection{Christianisme versus hellénisme}

Le texte de La Barbarie conduira naturellement aux réflexions plus tardives sur le christianisme. Henry entend repenser le statut phénoménologique du religieux dans la culture, dans l'optique générale du nihilisme comme crise de la modernité. Caractériser la religion comme procès phénoménologique de la venue de la vie en elle-même permet à Henry de fixer les conditions d'une sortie du nihilisme. Un texte intitulé «christianisme et phénoménologie » ${ }^{37}$, prononcé en 1996, à l'époque de la parution de C'est moi la Vérité, établit clairement le lien entre la réflexion sur la culture élaborée dans La Barbarie, et la nécessité de développer une philosophie du christianisme dix ans plus tard. Henry oppose clairement le christianisme à la modernité et à l'idéologie de la science, comprise comme autodestruction de la subjectivité en un sens phénoménologique.

On constate que la rupture avec Nietzsche a lieu, sans grande surprise, à ce moment précis. Henry évoque désormais la pensée du philosophe allemand en affirmant qu' « elle parle de la vie dans le monde moderne, mais en la défigurant ${ }^{38}$. Jusqu'alors présenté comme le héraut d'un concept anti-grec de la vie, Nietzsche est à présent rejeté au même titre que les autres penseurs de la tradition. La vie nietzschéenne devient un processus ontique absurde et aveugle de recherche de puissance, dont la modernité subirait les ravages. Henry rejoint ici un jugement quasi heideggérien. À peu près à la même époque, on constate que Henry reproche également à Nietzsche sa mécompréhension du christianisme. En interprétant cette religion comme une religion du mépris du corps, Nietzsche aurait manqué la distinction phénoménologique du corps et de la chair, et défiguré la foi

${ }^{37}$ Michel Henry, AD, p. 139-158.

${ }^{38}$ Ibid., p. 146. 
chrétienne. Selon Henry, le christianisme ne méprise par le charnel : «C’est une contrevérité, accréditée par Nietzsche ; en réalité, le christianisme promeut une conception grandiose de la chair. $\gg{ }^{39}$ L'ignorance nietzschéenne de la distinction du corps-objet et de la chair invisible permettrait alors de remettre en question l'association entre christianisme et nihilisme développée par Nietzsche dans les paragraphes 6 et 7 de l'Antéchrist. Henry ne conçoit pas la religion chrétienne comme une négation de la vie, mais au contraire comme un approfondissement de la venue en soi de la vie. Plus généralement, dès l'époque de La Barbarie, Henry définit la religion comme "l'épreuve [...] que la vie fait d'elle-même en ce Fond mystérieux d'où elle jaillit et ne cesse de s'étreindre soi-même ${ }^{40}$. Sur cette base, le christianisme sera pensé comme une praxis phénoménologique du retour à soi de la vie, destinée à vaincre l'oubli de la vie issu de l’idéologie de la science.

En quoi l'auto-affection peut-elle avoir un sens religieux et proprement chrétien ? Tout vivant est dans une passivité à l'égard de la vie, qui n'est autre que l'autoaffection première de la vie absolue, dans laquelle les vivants naissent lorsque que la vie leur est donnée. Aux yeux de Henry, la religion chrétienne est la praxis phénoménologique du retour à ce fond comme Salut, et l'homme doit faire retour à son essence comme Fils de Dieu. Tant que l'homme ignore ce retour à la vie infinie, son humanité est perdue ${ }^{41}$. Henry substitue donc à l'animal rationnel grec, ainsi qu'au Surhomme de Nietzsche, la théorie chrétienne du Fils, comme théorie de la seconde naissance ou de la ré-génération, dont il faut mesurer l'importance à l'aune du nihilisme de la modernité. La conclusion de C'est moi la vérité porte en effet sur « le christianisme et le monde moderne ». Henry suggère ici clairement qu'il faut lire cette philosophie du christianisme en lien avec l'analyse de la modernité dégagée dans La Barbarie. Or, Henry décrit à ce moment le monde moderne comme "l’Anti-Christ » ${ }^{42}$. Ce monde vient nier que Jésus soit le Christ, et dénonce ainsi l'existence d'un premier Soi généré dans l'auto-génération de la vie. En critiquant la notion d'Anti-Christ, Henry vise implicitement la pensée nietzschéenne comme nihilisme, et comme horizon non résolu de la crise de la modernité.

Au cœur de cette opposition sur la nature du christianisme, n'est-ce pas une divergence profonde qui se révèle entre Henry et Nietzsche ? La conception nietzschéenne de la vie n'est de toute évidence pas chrétienne, puisque le philosophe allemand ne pose jamais la vie comme une valeur absolue. Dans la Généalogie de la psychanalyse, Henry déplaçait déjà le sens du concept nietzschéen de valeur. Aux yeux du phénoménologue français, les valeurs procèdent de la vie, dans le sens où la vie est la source de toute évaluation - ce premier point n'est pas contestable d'un point de vue strictement nietzschéen - mais Henry franchit sur cette base un pas

\footnotetext{
${ }^{39}$ Michel Henry, Entretien accordé à Valeurs actuelles, 22 décembre 2000.

${ }^{40}$ Michel Henry, B, p. 3.

${ }^{41}$ Michel Henry, MV, p. 330.

${ }^{42}$ Cf. sur ce problème Michel Henry, MV, p. 336 et suivantes.
} 
que Nietzsche ne franchit jamais, en affirmant que la vie est la source de toute valeur, parce qu'elle est d'abord et avant tout la valeur absolue. Aux antipodes de cette pensée chrétienne, plus précisément de l'interprétation henryenne du christianisme, Nietzsche considère la valeur de la vie comme problématique. Aux yeux de Nietzsche, la philosophie peut penser la vie à condition d'abandonner toute valorisation inconditionnelle. La grande souffrance, dans laquelle l'homme aura patienté, enduré le désespoir, se sera lentement consumé, pourra seule permettre de regarder la vie avec l'œil aiguisé du philosophe : «La confiance dans la vie s'est évanouie : la vie elle-même est devenue problème. ${ }^{43}$ Chez Nietzsche, la nécessité de créer des valeurs doit être ramenée à ce cadre philosophique général de la vie comme problème axiologique.

Nietzsche conçoit que les grandes cultures prennent pour point de départ ce caractère problématique de la vie, pour bâtir leur œuvre sur ce fondement précaire. Les valeurs les plus élevées seront celles qui permettront à la vie de se vouloir ellemême en tant que problématique. Dans La naissance de la tragédie, Nietzsche reconnaît que les Grecs de l'époque tragique ont réussi à mettre en place, sur le fond de ce pessimisme premier, une culture pourtant capable de dire «oui ». Plus tard, dans la préface du Gai Savoir, il renverra à nouveau aux Grecs pour élucider la valeur supérieure de leur culture : "Ces Grecs ! Ils s’y connaissaient, pour ce qui est de vivre: chose pour laquelle il est nécessaire de s'arrêter courageusement à la surface, au pli, à la peau, d'adorer l'apparence, de croire aux formes, aux sons, aux mots, à toute l'Olympe de l'apparence ! Ces Grecs étaient superficiels... par profondeur ! ${ }^{44}$ Nietzsche construit ici un modèle apollinien de la Grèce tragique. Cette culture a su valoriser l'apparence, elle est parvenue à s'arrêter à la surface des choses comme condition d'une vie élevée au rang d'un style. Un tel paradigme esthétique de la vie s’oppose dès lors à l’Absolu religieux de Henry.

\footnotetext{
${ }^{43}$ Friedrich Nietzsche, Le Gai Savoir, trad. par P. Wotling, Paris, GF Flammarion, 1997, p. 31.

${ }^{44}$ Ibid., p. 33.
} 


\section{Conclusion : les puissances abyssales de la vie}

Ici se révèle la tension interne de la lecture henryenne de Nietzsche. Henry auraitil cherché à critiquer l'apollinisme de la pensée occidentale en s'appuyant paradoxalement sur le penseur le plus apollinien de la tradition ? Certes Nietzsche n'est pas exclusivement un penseur de la belle apparence esthétique, puisque cette dernière cache une profondeur, celle de l'activité pulsionnelle dionysiaque. Mais cette profondeur n'est justement pas un Fond, comme chez Henry, auquel l'homme pourrait faire retour pour trouver le Salut. La vie nietzschéenne se caractérise par les « abîmes " ${ }^{45}$, sans limites assignables, que l’homme peut traverser dans l'expérience de la souffrance. La surface apollinienne devient le seul support sur lequel la vie pourra s’appuyer, afin de ne pas sombrer dans les gouffres les plus inhospitaliers. La tension qui commande la lecture henryenne de Nietzsche peut alors être élucidée. Henry a tenté de réinvestir la critique nietzschéenne de la Grèce philosophique, mais n'est pas parvenu à donner un statut à la valorisation nietzschéenne de la Grèce tragique. L'opposition du fond et du sans-fond, du christianisme et de la Grèce archaïque, sépare définitivement Henry et Nietzsche, et remet en cause la lecture henryenne de Nietzsche, ce qui ne signifie pourtant pas que cette lecture soit dénuée de fécondité au sein de l’itinéraire de pensée du phénoménologue français.

Henry est lui aussi le penseur d'une « subjectivité abyssale » ${ }^{46}$, mais il convient de distinguer le Fond henryen et la profondeur nietzschéenne. Chez Henry, l'abyssal renvoie au fond de l'être, ou à l'être comme fond, qui n'est autre que la promesse du Salut. Sous la plume de Nietzsche, le retour au fond conduit au contraire à une perte de soi : « À force de vouloir aller au fond des choses, on tombe dans un puits sans fond. $"{ }^{47} \mathrm{Si}$ la vie ne repose sur rien, elle doit construire son équilibre dans le chaos, et tout horizon chrétien est ici dépassé au profit d’une pensée grecque de la vie comme œuvre d'art. Deux penseurs de la vie peuvent être très éloignés. Nietzsche serait-il, malgré les apparences, le plus grand rival de Henry ? Le phénoménologue de l'Absolu et le philosophe artiste pouvaient-ils se comprendre ? Ces deux penseurs sont pourtant ceux qui ont affronté le plus radicalement le problème du nihilisme, mais ils s'accuseraient l'un l'autre d'y succomber, au-delà de l'anachronisme qu'une telle affirmation implique. Il ne s'agit pas de donner raison à l'un plutôt qu'à l'autre. Dans cette confrontation, c'est un rapport de la vie à elle-même qui continue de se jouer. Est-ce un rapport nihiliste ? Cet horizon non résolu de la modernité est l'indice que la vie poursuit le mouvement par lequel elle se pense elle-même. Sa puissance dépasse son propre pouvoir de compréhension. La philosophie de la vie aujourd'hui ne peut être que l'accomplissement de cette expérience.

\footnotetext{
${ }^{45}$ Ibid., p. 31.

${ }^{46}$ Sur cette expression, cf. par exemple Michel Henry, VI, p. 24.

${ }^{47}$ Friedrich Nietzsche, Fragments Posthumes XIV, trad. par J-C. Hémery, Paris, Gallimard, 1977, FP 20 [73], p. 307.
} 
